\title{
Soft tissue reconstruction in wide Tessier number 3 cleft using the straight-line advanced release technique
}

Gyeong Hoe Kim',

Rong Min Baek², Baek Kyu Kim²

${ }^{1}$ Department of Plastic and

Reconstructive Surgery, Seoul National University Hospital, Seoul National University College of Medicine, Seoul; ${ }^{2}$ Department of Plastic and Reconstructive Surgery, Seoul National University Bundang Hospital, Seoul National University College of Medicine, Seongnam, Korea

\begin{abstract}
Craniofacial cleft is a rare disease, and has multiple variations with a wide spectrum of severity. Among several classification systems of craniofacial clefts, the Tessier classification is the most widely used because of its simplicity and treatment-oriented approach. We report the case of a Tessier number 3 cleft with wide soft tissue and skeletal defect that resulted in direct communication among the orbital, maxillary sinus, nasal, and oral cavities. We performed soft tissue reconstruction using the straight-line advanced release technique that was devised for unilateral cleft lip repair. The extension of the lateral mucosal and medial mucosal flaps, the turn over flap from the outward turning lower eyelid, and wide dissection around the orbicularis oris muscle enabled successful soft tissue reconstruction without complications. Through this case, we have proved that the straight-line advanced release technique can be applied to severe craniofacial cleft repair as well as unilateral cleft lip repair.
\end{abstract}

Keywords: Cleft lip / Cleft palate / Congenital abnormality / Craniofacial abnormality / Reconstructive surgical procedure

\section{INTRODUCTION}

Craniofacial cleft is an extremely rare congenital disease that presents facial and cranial disfigurement with various patterns and degrees of severity [1-3]. When using the Tessier classification, a number 3 cleft is the most common type, ranging from the mild type with coloboma only to the severe type involving both orbital, maxillary sinus, nasal, and oral cavities [2,4]. Although several surgical techniques have been reported for soft tissue reconstruction in number 3 clefts [5-8], there is no standard method; further, they are incompatible with other cleft repair techniques. Therefore, by applying a unilateral cleft repair

\footnotetext{
Correspondence: Baek Kyu Kim

Department of Plastic and Reconstructive Surgery, Seoul National University Bundang Hospital, Seoul National University College of Medicine, 82 Gumi-ro 173beon-gil, Bundang-gu, Seongnam 13620, Korea

E-mail: plasrecon@gmail.com

Received May 24, 2019 / Revised July 4, 2019 / Accepted July 5, 2019
}

method, we developed a simple and easily reproducible surgical technique for soft tissue reconstruction in a number 3 cleft.

\section{CASE REPORT}

A 15-month-old girl presented with a right-side congenital craniofacial cleft. She also had syndactyly at the third webspace of the right hand and the second and fourth webspace of the left foot, but she was never diagnosed with syndromic disorder and never had a genetic consultation. A physical examination revealed that the cleft originated from the lateral to right Cupid's bow and involved the lip, alveolus, palate, cheek, and lower eyelid (Fig. 1). The ipsilateral ala was displaced superiorly, but the circumferential shape of the nostril was intact. The exposed cornea had keratosis and she had severe dystopia and enophthalmos due to the absence of an inferior orbital wall. Preoperative computed tomography revealed that the cleft originated 


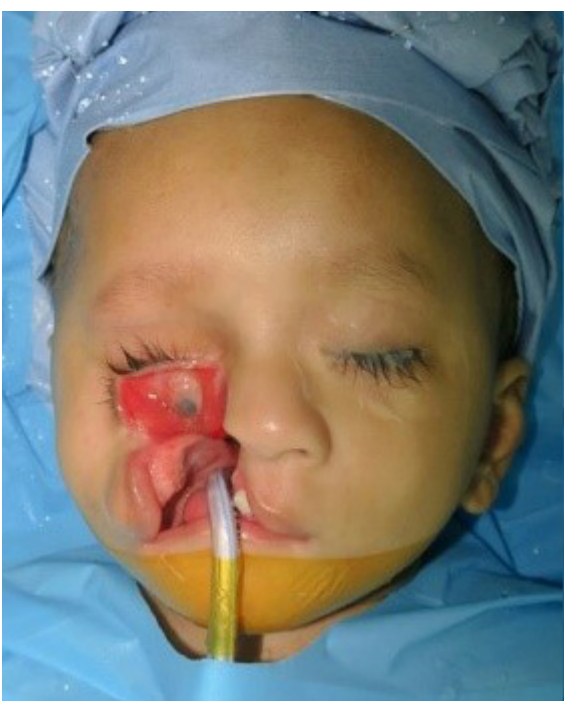

Fig. 1. Preoperative photograph of the patient. The cleft originated lateral to right of the Cupid's bow involving the lip, alveolus, palate, cheek, lower eyelid, and extended into the medial lower eyelid.

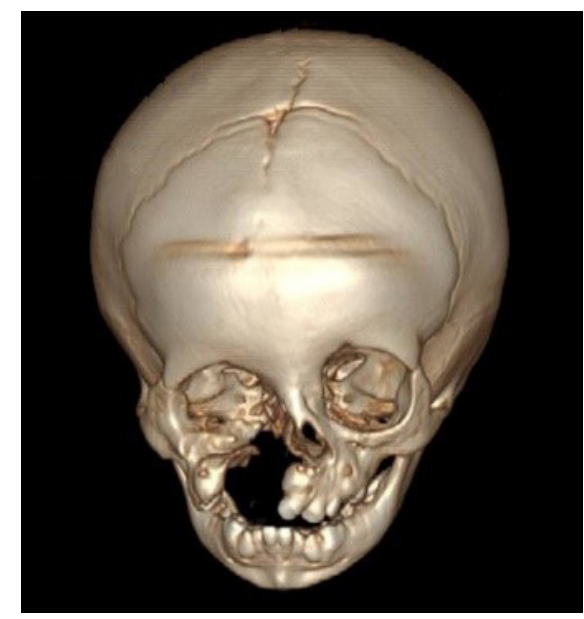

Fig. 2. Preoperative computed tomography of the patient. It showed a direct communication among the oral cavity, maxillary sinus, and nasal and orbital cavities.

from the lateral to ipsilateral central incisor involving the medial and inferior wall of the maxillary sinus and inferior orbital wall (Fig. 2). This resulted in direct communication among the orbital, maxillary sinus, nasal, and oral cavity. Thus, we concluded that this craniofacial cleft corresponded to the number 3 cleft according to the Tessier classification.

Under general anesthesia, we performed soft tissue reconstruction using the straight-line advanced release technique, which was developed for unilateral cleft lip repair (Fig. 3). Design of the lateral mucosal (L) flap was extended to turn outward from the lower eyelid. Because it was difficult to cover all the inner lining of the cheek with the $\mathrm{L}$ flap alone, the medial mucosal (M) flap was widely designed to include the philtral
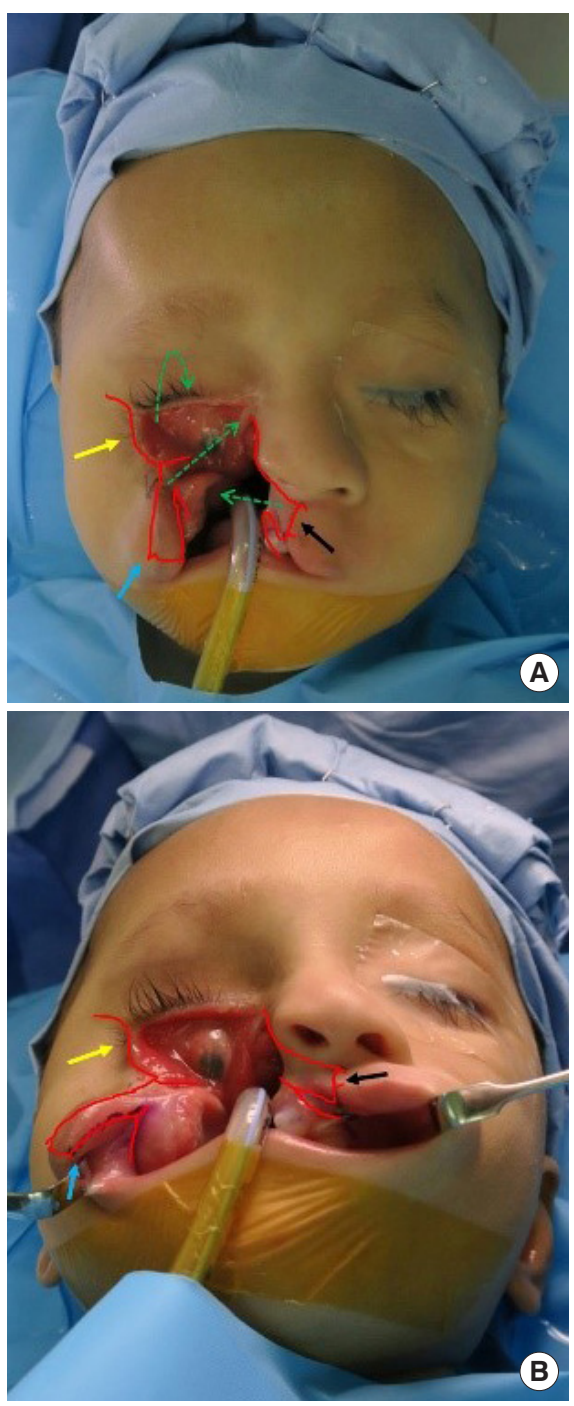

Fig. 3. (A, B) Preoperative design of the surgical method (yellow arrows, the turn-over flap for the lateral lower eyelid reconstruction; blue arrows, the lateral mucosal flap for the superior inner lining of the cheek; black arrows, the medial mucosal flap for inferior inner lining of the cheek; green dotted arrows, the direction of transposition of each flap).

tissue. The upper incision of the $\mathrm{M}$ flap was extended along the skin mucosal junction lateral to the nasofacial groove for the attachment of the $\mathrm{L}$ flap. As in the original technique, a triangular flap design and two slit incisions were also added in the design of the $\mathrm{L}$ and $\mathrm{M}$ flaps and the inner linings were marked through the gingivolabial sulcus. Finally, we designed a turn-over flap that turned outward from the lower eyelid along the subciliary incision line to cover the lateral lower eyelid defect.

On the cleft side, dissection was performed to separate the orbicularis oris muscle from the entire maxillary plane, and the extent of dissection was set as wide as possible to pull the cheek tissue maximally to the medial side. On the non-cleft side, the abnormally inserted orbicularis oris muscle was released from 


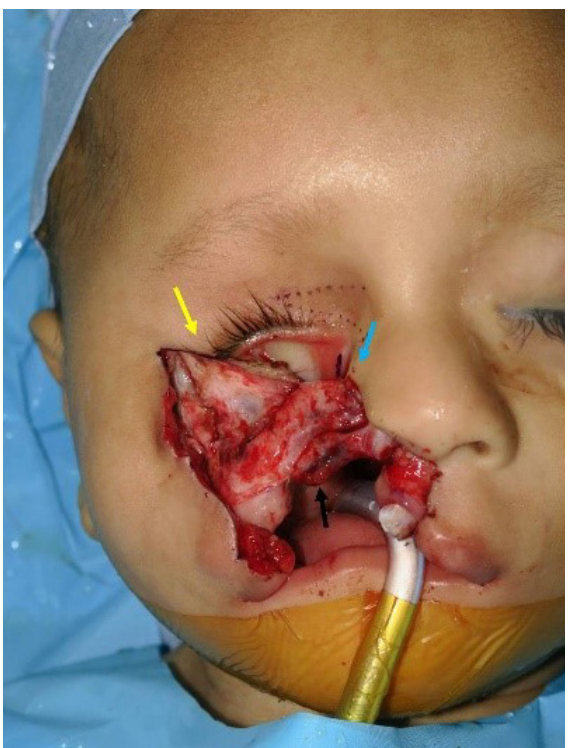

Fig. 4. Intraoperative photograph after complete reconstruction of the inner lining by interchanging the lateral and medial mucosal flaps and transposition of the turn-over flap from the lower eyelid (yellow arrow, the turn-over flap for lateral lower eyelid reconstruction; blue arrow, lateral mucosal flap for superior inner lining of the cheek; black arrow, medial mucosal flap for inferior inner lining of the cheek).

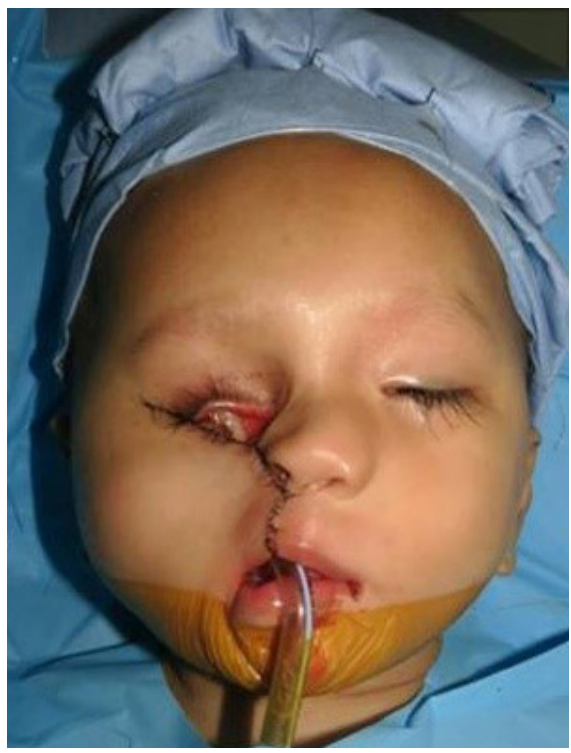

Fig. 5. Postoperative photograph showing complete soft tissue reconstruction of lip, cheek, and lower eyelid.

the anterior nasal spine and the base of pyriform aperture followed by subcutaneous dissection of the nasal dorsum and philtrum. After interchanging the $\mathrm{L}$ and $\mathrm{M}$ flap, the turn-over flap from the lower eyelid was added superiorly to complete the inner lining of the lower eyelid and cheek (Fig. 4). The freed orbicularis oris muscle was repaired with full width from the columellar base to the red vermilion. Finally, the oral lining of the

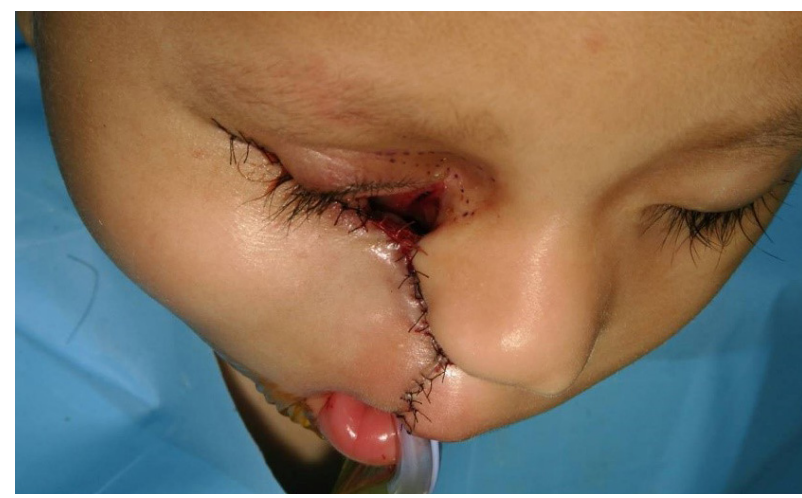

Fig. 6. Remnant medial lower eyelid defect forming the oroocular fistula after surgery. It needs to be repaired during a secondary surgery.

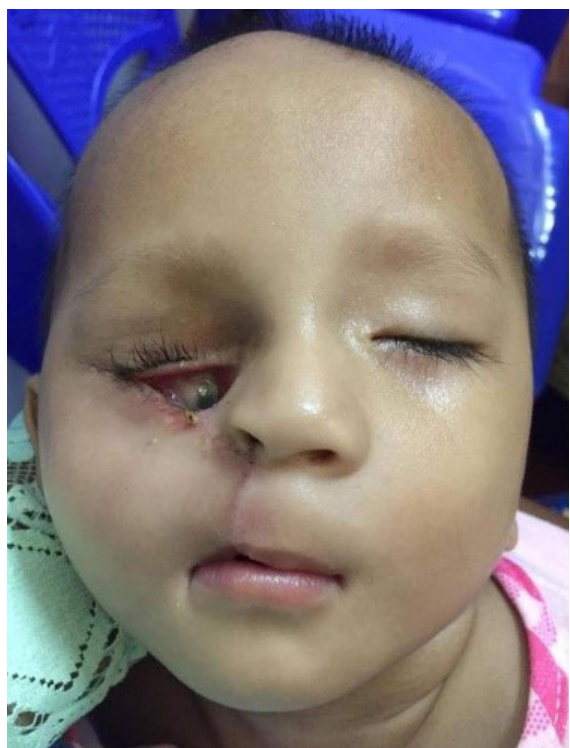

Fig. 7. Postoperative photograph of the patient 1 month after surgery. There was no wound dehiscence.

lip was reconstructed by advancing the bilateral oral lining flap that was elevated through the gingivolabial incision, and skin closure was completed by advancing bilaterally undermined skin. Because of the widely performed subcutaneous dissection, there was no high tension on the skin suture.

The postoperative photographs showed the complete soft tissue reconstruction of the lip, cheek, and lower eyelid (Fig. 5). However, a defect remained in the medial lower eyelid forming the oroocular fistula, which needed to be repaired during a secondary surgery (Fig. 6). On the first postoperative day, there was no flap congestion, and the patient was discharged. The stitches were removed from the wound on the 7th postoperative day and there was no wound dehiscence 1 month after surgery (Fig. 7). 


\section{DISCUSSION}

A craniofacial cleft, which is reported to occur sporadically in 1.4 to 4.9 in 100,000 live births [1], has one of the most disfigured appearances of all congenital facial anomalies $[2,3]$. The etiology of craniofacial clefts is explained through a failure in the fusion of the mesoderm during the embryonic process or constriction by amniotic bands [9]. Because of its variability and complexity, there are several classification systems, such as Karik classification, van der Meulen classification, and Tessier classification $[2,10,11]$. Among them, Tessier classification has been most widely used due to its simplicity and treatment-oriented approach, classifying craniofacial clefts from numbers 0 to 14 according to their anatomical position.

Among the Tessier craniofacial clefts, the number 3 cleft, first reported in 1887 [4], is the most common type. The number 3 cleft passes the philtral column and extends into the lower eyelid medial to the inferior punctum. The tissue deficiency between the alar base and the lower eyelid results in a shortened nose on the affected side. It also carries the disruption of the nasolacrimal system and the lower eyelid defect causes corneal erosion and loss of vision. In the skeletal system, the number 3 cleft involves the frontal process of the maxilla and orbital floor, which leads to direct communication among the orbital, maxillary sinus, nasal, and oral cavity. These features are all present in this case.

Several surgical techniques for craniofacial cleft repair have been reported through a small number of case reports. In 1985, van der Meulen [5] reported the surgical technique using the transposition of a forehead flap or rotation and advancement of a cheek flap. In 1996, Akoz et al. [6] reported the Z-plasty based method for the repair of a bilaterally involved craniofacial cleft. Longaker et al. [7] has used the superiorly based nasolabial flap for lower eyelid reconstruction during craniofacial cleft repair. On the other hand, Menard et al. [8] used a tissue expansion technique for craniofacial cleft repair to overcome the skin and soft tissue deficiency and achieve tension-free closure.

In this case, we were successful in soft tissue reconstruction of a wide craniofacial cleft by using the Straight-line advanced release technique. In fact, the Straight-line advanced release technique is a novel technique that was devised for unilateral cleft lip repair by Baek et al. [12]. Due to the simplicity of the surgical design, it has been widely performed by many plastic surgeons and even novice plastic surgeons in our hospital. Through this case, we have proved that the Straight-Line Advanced Release technique can be applied to a wide craniofacial cleft repair as well as unilateral cleft lip repair. The extension of the $\mathrm{L}$ and $\mathrm{M}$ flaps along the cleft margin, the inclusion of phil- tral tissue in the $\mathrm{M}$ flap, and the turn-over flap that turned outward from the lower eyelid enabled successful soft tissue reconstruction. Above all, due to the wide dissection around the orbicularis oris muscle, tension-free closure was possible.

Our surgical technique did not include reconstruction of the medial lower eyelid defect, leaving an oroocular fistula. Since this patient had a bony defect in the inferior orbital wall, soft tissue reconstruction alone could not sufficiently obliterate the oroocular fistula. Furthermore, due to the association of frequent lower lid medial coloboma relapses after soft tissue reconstruction alone in patients with wide tissue deficiency [13], we planned to perform the skeletal reconstruction and oroocular fistula repair simultaneously in the secondary surgery. Soft tissue reconstruction of the medial lower eyelid defect can be achieved with upper eyelid transposition flap or superiorly based nasolabial flap $[7,14]$. For skeletal reconstruction, a split calvarial bone graft will be the primary choice and a costochondral graft can be used if the defect is large [15]. The secondary surgery will occur after the patient is 3 years old, when the diploic bone is differentiated enough [16]. We expect to achieve complete reconstruction of the craniofacial cleft after the secondary surgery.

\section{NOTES}

\section{Conflict of interest}

No potential conflict of interest relevant to this article was reported.

\section{Ethical approval}

The study was approved by the Institutional Review Board of Seoul National University Bundang Hospital (IRB No. B-1096/ 544-701) and performed in accordance with the principles of the Declaration of Helsinki. Written informed consent was obtained.

\section{Patient consent}

The patient's parent provided written informed consent for the publication and the use of her images.

\section{ORCID}

Gyeong Hoe Kim https://orcid.org/0000-0001-5992-2091

Rong Min Baek https://orcid.org/0000-0002-0705-658X

Baek Kyu Kim https://orcid.org/0000-0001-9974-5165

\section{REFERENCES}

1. Kawamoto HK Jr. The kaleidoscopic world of rare craniofacial 
clefts: order out of chaos (Tessier classification). Clin Plast Surg 1976;3:529-72.

2. Tessier P. Anatomical classification facial, cranio-facial and latero-facial clefts. J Maxillofac Surg 1976;4:69-92.

3. Kawamoto HK Jr. Rare craniofacial clefts. In: McCarthy JG, editor. Plastic surgery. Philadelphia: Saunders; 1990. p. 2922-73.

4. Morian R. Ueber die schrage Gesichtsspalte. Arch Klin Chir 1887;35:24.

5. van der Meulen JC. Oblique facial clefts: pathology, etiology, and reconstruction. Plast Reconstr Surg 1985;76:212-24.

6. Akoz T, Erdogan B, Gorgu M, Kutlay R, Dag F. Bilaterally involved Tessier no. 4 cleft: case report. Cleft Palate Craniofac J 1996;33:252-4.

7. Longaker MT, Lipshutz GS, Kawamoto HK Jr. Reconstruction of Tessier no. 4 clefts revisited. Plast Reconstr Surg 1997;99: 1501-7.

8. Menard RM, Moore MH, David DJ. Tissue expansion in the reconstruction of Tessier craniofacial clefts: a series of 17 patients. Plast Reconstr Surg 1999;103:779-86.

9. Sperber GH. Craniofacial development. Hamilton: BC Decker;
2001.

10. Karik V. Proposed classification of rare congenital cleft malformations in the face. Acta Chir Plast 1966;8:163-8.

11. van der Meulen JC, Mazzola R, Vermey-Keers C, Stricker M, Raphael B. A morphogenetic classification of craniofacial malformations. Plast Reconstr Surg 1983;71:560-72.

12. Baek RM, Choi JH, Kim BK. Practical repair method for unilateral cleft lips: straight-line advanced release technique. Ann Plast Surg 2016;76:399-405.

13. Dey DL. Oblique facial clefts. Plast Reconstr Surg 1973;52:25863.

14. Tessier P, Rougier J, Hervouet F, Woilllez M, Lekieffre M, Demore P. Plastic surgery of the orbits and eyelids. Chicago: Masson Publishing; 1981.

15. Allam KA, Lim AA, Elsherbiny A, Kawamoto HK. The Tessier number 3 cleft: a report of 10 cases and review of literature. J Plast Reconstr Aesthet Surg 2014;67:1055-62.

16. Koenig WJ, Donovan JM, Pensler JM. Cranial bone grafting in children. Plast Reconstr Surg 1995;95:1-4. 